\title{
ZIKA Virus Evades Host Innate Immunity through its Non-structural Proteins
}

\section{Yancui Wang ${ }^{1}$, Limin Chen ${ }^{1,2}$ and Yujia $\mathbf{L i}^{\mathbf{1}^{\star}}$}

${ }^{1}$ Provincial Key Laboratory for Transfusion-Transmitted Infectious Diseases, Institute of Blood Transfusion, Chinese Academy of Medical Sciences and Peking Union Medical College, Chengdu, Sichuan, China

${ }^{2}$ Toronto General Research Institute, University of Toronto, Toronto, Canada

\section{Brief Introduction to Zika Virus (ZIKV)}

Zika virus (ZIKV) is an emerging mosquito-borne pathogen that was initially isolated from a rhesus monkey in Uganda in 1947 [1-3]. ZIKV infection is associated with Guillain-Barre syndrome in adults and other neurological defects in new-borns [4-6] which prompted the World Health Organization (WHO) to declare a Public Health Emergency of International concern on February 1, 2016 [7]. There are no proved vaccines or drugs to prevent or treat ZIKV infection. Hence, there is a pressing need to study the molecular pathogenesis of ZIKV to facilitate the development of effective vaccines and antiviral therapies.

\section{The Genome Structure of ZIKV}

ZIKV is a single-stranded positive RNA genome of flavivirus whose genome consists of about 11,000 nucleotides with two flanking non-coding regions and a single long open reading frame (ORF), which encodes three structural proteins: the capsid(C), premembrane/ membrane (prM/M), and envelope (E), and seven non-structural proteins (NS1, NS2A, NS2B, NS3, NS4A, NS4B, and NS5) which participate in RNA replication [8]. Structural protein $C$ interacts with the viral RNA to form a nucleocapsid while structural protein prM inhibits premature viral fusion with host membranes. The function of structural protein $\mathrm{E}$ is to regulate cellular attachment and to accelerate membrane fusion, virus assembly and release the viral genome into the host $[9,10]$. Nonstructural proteins mediate viral transcription and replication and also engage in mitigating host antiviral responses to facilicate ZIKV replication [6,10].

\section{Mechanism of Non-Structural Proteins of ZIKV in Evading Host Innate Immunity}

Innate and adaptive immune responses were mounted to restrict viral RNA replication and virus spread of mosquito-borne flaviviruses. ZIKV replication was significantly inhibited in cells pre-treated with the dsRNA analogue, poly (I: C) or IFNs. However, ZIKV has developed effective countermeasures to inhibit the host innate immune response [11]. ZIKV specifically several of its non-structural proteins inhibited induction of a luciferase reporter gene expression driven by the IFN- $\beta$ promoter when stimulated with poly (I: C) or agonists of the RIG-Ilike helicase pathway. For example ZIKV impairs the induction of type I IFNs through binding to IFN regulatory factors (IRF3) $[12,13]$ and IFN- $\alpha / \beta$ receptor (INFAR) to mitigate the downstream signalling $[4,14]$.

Recent studies demonstrated that NS1 and NS4B proteins of ZIKV could bind to TBK1 and blocked TBK1 oligomerization, resulting in inhibiting type I IFN production at TBK1 level, whereas NS2BNS3 could interact with endogenous Jak1. Overexpression of NS2B3 markedly reduced the protein levels of Jak1, which consequently inhibited the phosphorylation of Jak1 and STAT1, leading to the suppression of Jak-STAT signalling to inhibit the IFN-mediated induction of interferon stimulated genes (ISGs). Meanwhile, NS2B3 of ZIKV also inhibited apoptosis, further facilitating virus replication.
Although type I IFNs restricted ZIKV replication by promoting the autophagic degradation of NS2B3, NS1 and NS4B proteins inhibited type I IFN production to stabilize NS2B3 [11,15]. Further study indicated that the NS2B3 protease of ZIKV could species-specifically impair the responses to agonists of the cGMP-AMP synthase/stimulator of IFN genes (cGAS/STING) signalling pathway in human but not in murine cells [16]. Like DENV, ZIKV NS5 interacted with STAT2, resulting in proteasomal degradation of the IFN-regulated transcriptional activator STAT2 from humans but not from mice $[11,17,18]$, which differs from the mechanism of dengue virus (DENV) NS5 [19]. ZIKV did not require the E3 ubiquitin ligase UBR4 to induce STAT2 degradation during viral infection $[17,20]$. Another study demonstrated that nonstructural proteins of ZIKV inhibited RIGI-induced IFN- $\beta$ production through distinct mechanisms: IFN- $\beta$ mRNA induction was required for the phosphorylation of TBK1, IKKe, and IRF3 [21]. NS2A, NS2B, and NS4B suppress TBK1 phosphorylation while NS4A inhibits IRF3 phosphorylation; and NS5 binds to IRF3 to form NS5/IRF3 complex to inhibit IRF3 phosphorylation. Mechanistically, NS1 mutation promotes the binding of NS1 to TBK1, resulting in reduced levels of TBK1 phosphorylation and IFN- $\beta$ expression [22].

\section{Conclusion}

Taken together, non-structural proteins of ZIKV, such as NS1, NS4B, NS2B3 and NS5 negatively modulated antiviral response at various levels by inhibiting host innate immune response to produce type I IFNs. Therefore, non-structural proteins of ZIKV provide potential strategies to target innate antiviral immunity, and all these data shed light on how ZIKV evades host innate immunity.

\section{References}

1. Faye O, Freire C C, lamarino A, Faye O, de Oliveira JVC, et al. (2014) Molecular evolution of Zika virus during its emergence in the $20^{\text {th }}$ century. PLoS Negl Trop Dis 8: e2636.

2. Ndeffo-Mbah ML, Parpia AS, Galvani AP (2016) Mitigating Prenatal Zika Virus Infection in the Americas. Ann Intern Med 165: 551-559.

3. Zhou H, Eaton B, Hu Z, Arif B (2016) Accidental discovery and isolation of Zika virus in Uganda and the relentless epidemiologist behind the investigations. Virol Sin 31: 357-361.

*Corresponding author: Yujia Li, Provincial Key Laboratory for TransfusionTransmitted Infectious Diseases, Institute of Blood Transfusion, Chinese Academy of Medical Sciences and Peking Union Medical College, Chengdu, Sichuan, China, Tel: 86-28-61648530; E-mail: lily83630@163.com

Received July 16, 2018; Accepted July 18, 2018; Published July 25, 2018

Citation: Wang Y, Chen L, Yujia Li (2018) ZIKA Virus Evades Host Innate Immunity through its Non-structural Proteins. J Bioanal Biomed 10: 96-97. doi:10.4172/1948593X.1000e156

Copyright: (C2018 Wang Y et al. This is an open-access article distributed under the terms of the Creative Commons Attribution License, which permits unrestricted use, distribution, and reproduction in any medium, provided the original author and source are credited. 
Citation: Wang Y, Chen L, Yujia Li (2018) ZIKA Virus Evades Host Innate Immunity through its Non-structural Proteins. J Bioanal Biomed 10: $96-97$. doi:10.4172/1948-593X.1000e156

4. de Araujo TVB, Martelli CT, de Souza WV, Rodrigues LC (2016) Zika virus and microcephaly - Authors' reply. Lancet Infect Dis 16: 1332

5. Liang Q, Luo Z, Zeng J, Chen W, Foo SS, et al. (2016) Zika Virus NS4A and NS4B Proteins Deregulate Akt-mTOR Signaling in Human Fetal Neural Stem Cells to Inhibit Neurogenesis and Induce Autophagy. Cell Stem Cell 19: 663671.

6. Lazear HM, Diamond MS (2016) Zika Virus: New Clinical Syndromes and Its Emergence in the Western Hemisphere. J Virol 90: 4864-4875.

7. Heymann DL, Hodgson A, Sall AA, Freedman DO, Staples JE, et al. (2016) Zika virus and microcephaly: why is this situation a PHEIC? Lancet 387: 719-721.

8. Ming GL, Tang H, Song H (2016) Advances in Zika Virus Research: Stem Cell Models, Challenges, and Opportunities. Cell Stem Cell 19: 690-702.

9. Lazear HM, Diamond MS (2016) Zika Virus: New Clinical Syndromes and Its Emergence in the Western Hemisphere. J Virol 90: 4864-4875.

10. Mukhopadhyay S, Kuhn RJ, Rossmann MG (2005) A structural perspective of the flavivirus life cycle. Nat Rev Microbiol 3: 13-22.

11. Wu Y, Liu Q, Zhou J, Xie W, Chen C, et al. (2017) Zika virus evades interferonmediated antiviral response through the co-operation of multiple nonstructural proteins in vitro. Cell Discov 3: 17006.

12. Dowd KA, DeMaso CR, Pelc RS, Speer SD, Smith ARY, et al. (2016) Broadly Neutralizing Activity of Zika Virus-Immune Sera Identifies a Single Viral Serotype. Cell Rep 16: 1485-1491.

13. Chaudhary V, Yuen KS, Chan JF, Chan CP, Wang PH, et al. (2017) Selective Activation of Type II Interferon Signaling by Zika Virus NS5 Protein. J Virol 91: e00163-17.
14. Bowen JR, Quicke KM, Maddur MS, O'Neal JT, McDonald CE, et al. (2017) Zika Virus Antagonizes Type I Interferon Responses during Infection of Human Dendritic Cells. PLoS Pathog 13: e1006164.

15. Diamond MS (2009) Mechanisms of evasion of the type I interferon antiviral response by flaviviruses. J Interferon Cytokine Res 29: 521-530.

16. Ding Q, Gaska JM, Douam F, Wei L, Kim D (2018) Species-specific disruption of STING-dependent antiviral cellular defenses by the Zika virus NS2B3 protease. Proc Natl Acad Sci U S A 115: E6310-E6318.

17. Jones M, Davidson A, Hibbert L, Gruenwald P, Schlaak J, et al. (2005) Dengue virus inhibits alpha interferon signaling by reducing STAT2 expression. J Viro 79: 5414-5420.

18. Grant A, Ponia S S, Tripathi S, Balasubramaniam V, Miorin L, et al. (2016) Zika Virus Targets Human STAT2 to Inhibit Type I Interferon Signaling. Cell Host Microbe 19: 882-890.

19. Mazzon M, Jones M, Davidson A, Chain B, Jacobs M (2009) Dengue virus NS5 inhibits interferon-alpha signaling by blocking signal transducer and activator of transcription 2 phosphorylation. J Infect Dis 200: 1261-1270.

20. Morrison J, Laurent-Rolle M, Maestre AM, Rajsbaum R, Pisanelli G, et al (2013) Dengue virus co-opts UBR4 to degrade STAT2 and antagonize type I interferon signalling. PLoS Pathog 9: e1003265.

21. Kato H, Takeuchi O, Sato S, Yoneyama M, Yamamoto M, et al. (2006) Differential roles of MDA5 and RIG-I helicases in the recognition of RNA viruses. Nature 441: 101-105

22. Xia H, Luo H, Shan C, Muruato AE, Nunes BTD, et al. (2018) An evolutionary NS1 mutation enhances Zika virus evasion of host interferon induction. Nat Commun 9: 414. 